\title{
Biochemical changes in Non-alcoholic fatty liver disease (NAFLD): A study in Nepalese Population Pooja Maharjan ${ }^{1^{*}}$,PuspaRaj Khanal ${ }^{1 *}$,Narayan Prasad Parajuli ${ }^{1}$, Govardhan Joshi ${ }^{1}$, Hridaya Parajuli ${ }^{1}$, Santosh Khanal ${ }^{2}$, Bibek Bhatta ${ }^{3}$, Dipendra Raj Pandeya ${ }^{4}$
}

\author{
Affiliations: \\ ${ }^{1}$ Department of Laboratory Medicine, Manmohan Memorial \\ Institute of Health Sciences, Kathmandu, Nepal \\ ${ }^{2}$ Department of Clinical Biochemistry, Manmohan Memorial \\ Community Hospital, Kathmandu, Nepal \\ ${ }^{3}$ Department of Clinical Microbiology, Nobel College, \\ Kathmandu, Nepal \\ ${ }^{4}$ Department of Biochemistry, College of Medicine, Nepalese \\ Army Institute of Health Sciences, Kathmandu, Nepal \\ *These authors have contributed equally in this manuscript
}

Correspondence to:

Dr. Dipendra Raj Pandeya

Department of Biochemistry,

College of Medicine,

Nepalese Army Institute of Health Sciences, Kathmandu

Email: dipendra.pandey@gmail.com

How to cite this article:

Maharjan P, Khanal PR, Parajuli NP, Joshi G, Parajuli H, Khanal S, Bhatta B, Pandeya DR. Biochemical changes in non-alcoholic fatty liver disease (NAFLD): A study in Nepalese population. Ann. Clin. Chem. Lab. Med. 2016:2(2);15-20

DOI: http://dx.doi.org/10.3126/acclm.v2i2.15597

(C) 2016 Nepalese Association for Clinical Chemistry

\section{(c) (1) (2)}

This work is licensed under a Creative Commons AttributionShareAlike 4.0 International License.

\section{Abstract}

BACKGROUND

Non-alcoholic fatty liver disease (NAFLD) has emerged as the most common liver problem in the western world and is a clinicopathologic entity increasingly recognized as a major health burden in developed countries. Different laboratory tests are extremely useful in achieving a better understanding of diseases, and thereby, allow making decision for better management. The examination of different biochemical parameters usually provides excellent clues to the cause of the disease. The present study was conducted with the aim to assess the biochemical markers in Non alcoholic fatty liver disease (NAFLD) patients in Nepalese population.

METHODS

The biochemical parameters were investigated in 75 NAFLD patients, and 70 normal participants. The diagnosis of hepatic steatosis was established by abdominal ultrasound examination. All patients diagnosed as NAFLD were investigated for biochemical parameters and see the relationship between NAFLD and control was studied.

RESULTS

The findings of all biochemical parameters were raised in NAFLD patients in comparison with nonfatty liver control group and the differences were found to be statistically ( $P$ value less than 0.005 ) significant.

\section{CONCLUSIONS}

NAFLD is associated with changes in biochemical parameters in cases of NAFLD. Its early detection will help in modifying the disease course, delaying complications and will also play a major role in preventive cardiology.

KEYWORDS: Nonalcoholic Fatty Liver Disease (NAFLD), Lipid profile, Liver function test (LFT), Biomarker 


\section{Introduction}

Non-alcoholic fatty liver disease (NAFLD) is an appalling public health problem. It is considered the most common chronic liver disease in affluent societies, affecting $2-10 \%$ of the general population, encompassing a wide range of diseases from simple steatosis to nonalcoholic steatohepatitis (NASH) in both children and adults[1,2]. The liver cell damage that is observed in patients with NASH can lead to cirrhosis or even end-stage liver disease $[3,4]$. Absence of sign and symptoms, and deficiency of sensitive and specific diagnostic tests, limits the ability to estimate the prevalence of NAFLD. It has also been observed that with modernization, a sedentary lifestyle and a lack of exercise are tied in with an increased prevalence of diabetes mellitus (DM), obesity, hypertension and hypertriglyceridemia. These are considered to be important causes of nonalcoholic fatty liver disease (NAFLD). In the majority of patients, NAFLD is associated with metabolic risk factors such as obesity, diabetes mellitus, and dyslipidemia. Approximately 20$25 \%$ of the cases progress into cirrhosis with all its ramifications including hepatocellular carcinoma and the need for liver transplantation.

Biochemical tests are extremely useful for accomplishing a better understanding of the disease, and thereby allows thoughtfulmanagement decisions to be made. An ultrasound examination ofthe liver has relatively high sensitivity (60-95\%) and specificity (88-95\%)[5]. Therefore, the diagnosis of NAFLD has been made on liver ultrasound examination and the measurement of different biochemical parameters indicating liver injury or damage. Conflating the results into various scores may fortify the diagnosis [6]. However, researchers are still looking for simple diagnostic tools with greater sensitivity and specificity that could serve as a screening test for excessive fat accumulation in the liver.The data indicate that NAFLD may have male preponderance or an equal gender distribution and may even occur in the absence of diabetes and obesity [7].NAFLD can occur at all ages including childhood, though the highest prevalence is described in those between 40-50 years of age. With some limitations, both population and hospital-based studies from the west report that around 10-24 percent ecumenical population and 57-75 percent obese individual may have NAFLD [8].Thus, in view of the above contest, the present study was under taken to access the correlation of NAFLD with different biochemical parametersespecially Liver Function Tests (LFT) and Lipid profile tests in Nepalese population.

\section{Methods}

\section{Patients' characteristics}

Seventy five Patients were enrolled in the current study. They were selected from the outpatient clinics of Internal Medicine department, Manmohan Memorial Community Hospital, Kathmandu, Nepal. Patients' consent was obtained according to the regulations of the Nepalese Ministry of Health and the study design was approved by the local ethics committee.

\section{Diagnosis of NAFLD}

Nonalcoholic Fatty Liver Disease was diagnosed by noninvasive imagining liver ultrasoundprocedure. In addition, blood tests were performed to assess liver function and to exclude other causes of liver disease. The exclusion of significant alcohol intake was essential. Presence of abnormal fat accumulation in the liver found by $\mathrm{X}$-rays and ultrasound images confirmed the diagnosis.

The Inclusion criteria for all participants were:

(a) age between 20-70 years (b) newly diagnosis of NAFLD (c) good general health condition.

\section{The exclusion criteria were:}

(a) history of alcohol ingestion (b) malignancy (c) previous gastrointestinal tract surgery (d) smoking (e) presence of any liver disease that can cause fatty liver such as chronic hepatitis $C$, autoimmune liver disease (f) ingestion of drugs known to produce hepatic steatosis in the previous 6 months. 


\section{Statistical analysis}

The statistical analysis was under taken using SPSS version 20.0 software. All values are expressed as mean $\pm S D$. Student t- test was used to estimate the significant difference between the groups. The level of significance was considered when $p$ value $<0.05$.

\section{Results}

A total of 75 cases of NAFLD and 70 controls were included in this study. There were significant difference among control and NAFLD group considering age, the average age of the study group was $43.61 \pm 12.43$ years and $39.85 \pm 11.54$ years in control. Maximum numbers of patients were in $3^{\text {rd }}$ and $4^{\text {th }}$ decades of their life shownin the figure 1.

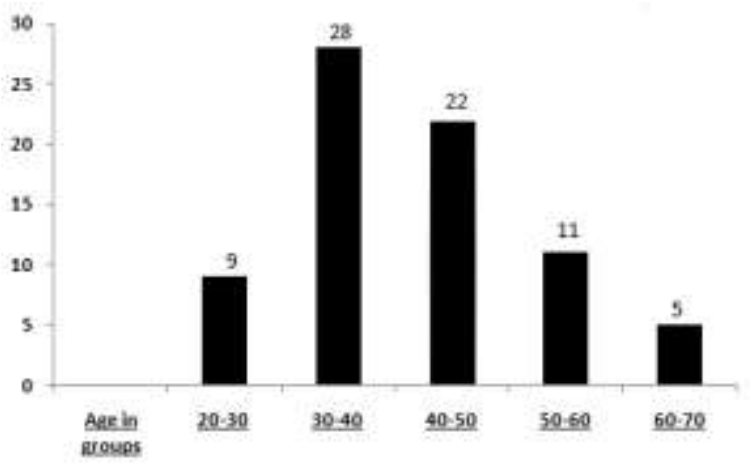

Figure 1:Age-wise distribution of patients with Non alcoholic fatty liver disease

The biochemical parameters including lipid profile and liver function tests (LFT) of all the cases of NAFLD and control groups are depicted in the table 1. In the present study, the average of all three markers (AST, ALT and ALP) increased significantly when comparison with control groups (Figure 2). Elevated level of AST, ALT and ALP levels were noticed in 53.8\%, $40.3 \%$ and $58.2 \%$ cases, respectively. As it is shown, the mean \pm SD of serum AST, ALT and ALP (IU/L) were 80.81+54.79, 70.63+42.58 and $231.78 \pm 150.92$ were significantly higher in NAFLD patients as compared to control group AST $(21.63 \pm 5.13)$, ALT $(23.67 \pm 6.01)$ and ALP $(124.33 \pm 29.60) p<0.005$.

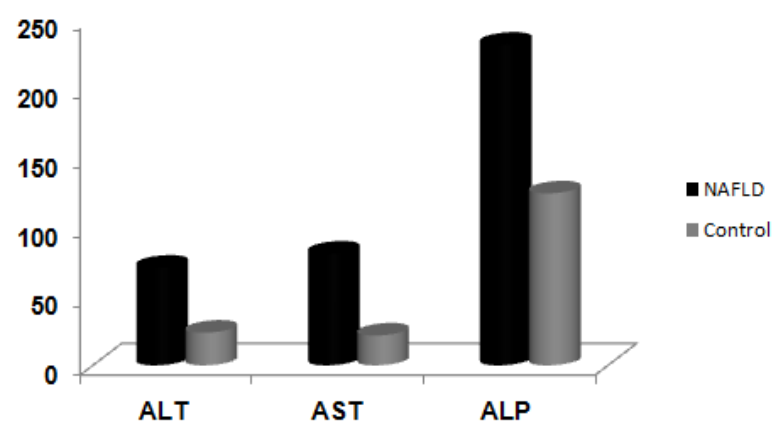

Figure 2: Comparison of Liver Function Tests (LFT) between NAFLD and control group

However, hyperlipidemia in $69.4 \%$ cases were observed. All the lipid profile parameters $(\mathrm{mg} / \mathrm{dl}) \quad$ total cholesterol $(228.06 \pm 22.37$; $\mathrm{p}<0.005)$, triglyceride(TG) $p<0.005), \operatorname{VLDL}(38.91 \pm 12.3 ; p<0.005)$ and LDL $(153.34 \pm 47.07 ; \quad(p<0.005) \quad$ were significantly higher in NAFLD patients as compared to non NAFLD group (Figure 3) total cholesterol

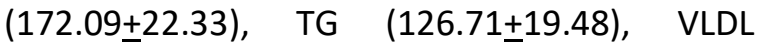
(25.38+16.11) and $\operatorname{LDL}(105.74 \pm 19.51)$. Paradoxically higher HDL $(40.91 \pm 9.95$ Vs $35.86 \pm 3.79 ; p<0.005)$ was seen on non-NAFLD group. 
Table 1: Comparison of Biochemical Parameters in Control and NAFLD

\begin{tabular}{llllc}
\hline S.No. & Variable & Control (Mean+SD) & NAFLD(Mean+SD) & P-Value \\
\hline 1 & Age & $39.85 \pm 11.54$ & $43.61 \pm 12.43$ & $<0.005$ \\
2 & Total Cholesterol(mg/dl) & $172.09 \pm 22.33$ & $228.06 \pm 2253.37$ & $<0.005$ \\
3 & Triglyceride $(\mathrm{mg} / \mathrm{dl})$ & $126.71 \pm 19.48$ & $193.57 \pm 99.41$ & $<0.005$ \\
4 & HDL Cholesterol $(\mathrm{mg} / \mathrm{dl})$ & $40.91 \pm 9.95$ & $35.86 \pm 3.79$ & $<0.005$ \\
5 & VLDL $(\mathrm{mg} / \mathrm{dl})$ & $25.38+16.11$ & $38.91 \pm 12.3$ & $<0.005$ \\
6 & LDL Cholesterol $(\mathrm{mg} / \mathrm{dl})$ & $105.74 \pm 19.51$ & $153.34 \pm 47.07$ & $<0.005$ \\
7 & ALT $(\mathrm{IU} / \mathrm{L})$ & $23.67 \pm 6.01$ & $70.63 \pm 42.58$ & $<0.005$ \\
8 & AST $(\mathrm{IU} / \mathrm{L})$ & $21.63 \pm 5.13$ & $80.81 \pm 54.79$ & $<0.005$ \\
9 & ALP $(\mathrm{IU} / \mathrm{L})$ & $124.33 \pm 29.60$ & $231.78 \pm 150.92$ & $<0.005$ \\
\hline
\end{tabular}

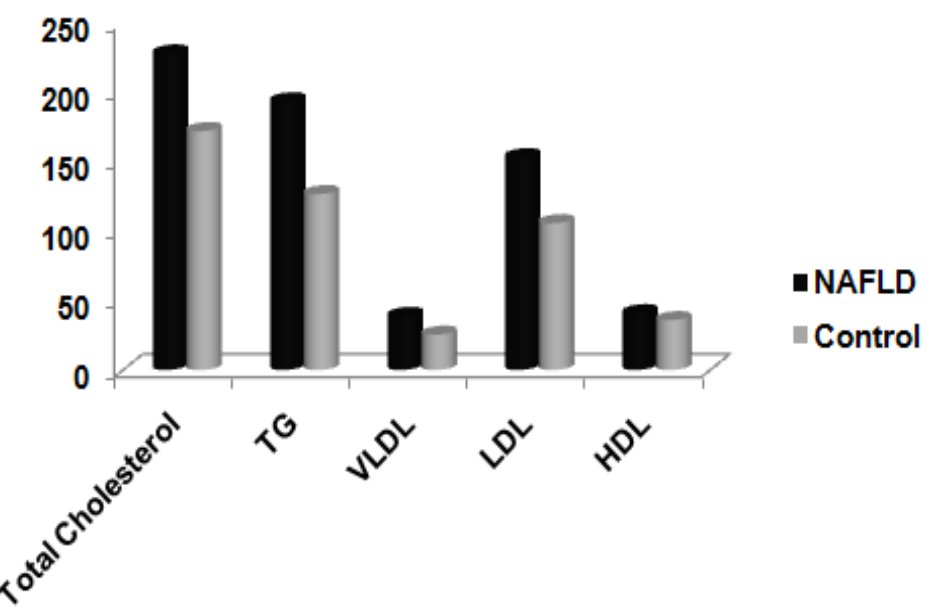

Figure 3: Comparison of Lipid Profile between NAFLD and control group

\section{Discussion}

Although NAFLD has emerged as the commonest liver problemworldwide and is found in up to one-third of the generalpopulation, $[9,10]$ there is little information on the true profileof patients with NAFLD in clinical practice. It has been well established that many biochemical abnormalities occur in chronic liver diseases. A number of studies have shown that NAFLD patients have significant increase in TG, total cholesterol, VLDL and LDL cholesterol, where as decreased HDL was noticed. Similarly deranged
AST, ALT and ALP were observed in greater percentages in patients of NAFLD than those without NAFLD. The results of our study also support these observations. As is seen, increase in all the above mentioned biomarkers, which can be very important in treatment aspect. The recorded all three above markers levels changes were statistically significant.

In Nepalese population, the subjects with NAFLD had significantly higher values of triglycerides $(193.57+99.41)$, total cholesterol $(228.06+2253.37)$, LDL $(153.34+47.07)$, and VLDL $(38.91+12.3)$, where as significantly lower HDL $(35.86+3.79)$ values were found than 
controls and the variances between them were shows significant $(P$ value $<0.005$ ) values. Bajaj et al[11] had also reported that the subjects with NAFLD had significantly higher values of total cholesterol and serum triglycerides. Increased lipid profile among NAFLD subjects had been reported in many studies. Clark et al[12] in USA in a cross-sectional study found that NAFLD subjects were higher in high triglycerides levels. In another cross]-sectional study in Brazil, subjects with NAFLD had a higher triglyceride[13]. However, LizardiCervera et al [14] in Mexico found that the high level of cholesterol was found in 63 percent of the NAFLD subjects.Similar findings were found also by other researchers $[15,16]$. Of note, the increased in TG in the patients was associated with insulin resistance. However, insulin resistance leads to hepatocytes fat deposition by two pathways; lipolysis and hyperinsulinemia [17].Insulin resistance is recognized as a major determinant of steatogenisis and possibly of liver disease progression. We have observed abnormal values of serum AST, ALT and ALP levels in all NAFLD groupthan those without NAFLD.75\% and 63\% (ALT and AST) had deranged in NAFLD group and a similar findingas noticed by Bajaj et al and Ajay Duseja et al $[18,19]$ respectively.Many studies during the recent years revealed that liver enzymes including AST and ALT can be useful to detect or predict NAFLD and its grading, but due to the high variability of liver enzymes, it does not seem that these enzymes have a definite role in the accurate diagnosis of fatty liver. Mikako

\section{REFERENCES}

1. Nobili V, Svegliati-Baroni G, Alisi A, Miele L, Valenti L, Vajro P. A 360-degree overview of paediatric NAFLD: recent insights. J Hepatol. 2013; 58(6):1218-29.

2. Kawano $Y$, Cohen DE. Mechanisms of hepatic triglyceride accumulation in non-alcoholic fatty liver disease. J Gastroenterol. 2013;48(4):434-41.

3. McCullough AJ. The clinical features, diagnosis and natural history of nonalcoholic fatty liver disease. Clin Liver Dis. 2004;8(3):521-33.
Obika also showed that liver enzymes do not appear to have any association with diagnose of NAFLD [20].

This Nepalese study showed that the majority of the patients were males, with the mean age of forty. This result is similar with different population base studies[21]. The ethnic differences were not included in this study. In Nepal, due to different caste/ethnic group recognized, it is an important subject to be investigated in NAFLD patterns in these groups. NAFLD is a multifactorial disease and many causes are related to the lifestyle, however, genetic predisposition also play a important role in the development of NAFLD. A limitation of our study is that the diagnosis of NAFLD was based on ultrasonography and was not confirmed by liver biopsy. Ultrasonography is by far the commonest method of diagnosing NAFLD in clinical practice.

\section{Conclusions}

In conclusion our study revealed that there is deranged lipid profile and liver function tests in casesof NAFLD group. Higher values of these biochemical parameters play an important role in increasing the severity and progression of the disease. Early detection would help not only in modifyingthe disease course and also delaying its further complications.

\section{Conflict of interest}

None declared.
4. Said A. Non-alcoholic fatty liver disease and liver transplantation: outcomes and advances. World J Gastroenterol. 2013;19(48):9146-55.

5. Charatcharoenwitthaya $P$, Lindor KD. Role of radiologic modalities in the management of non-alcoholic steatohepatitis. Clin Liver Dis. 2007; 11:37-54.

6. Poynard T, Ratziu V, Naveau S, Thabut D, Charlotte $F$, Messous $D$, et al. The diagnostic value of biomarkers (Steato Test) for the prediction of liver steatosis. Comp Hepatol. 2005;23(4):10. 
7. Sanyal AJ.Aga Technical review on nonalcoholic fatty liver disease. Gastroenterology.2002;123:1705-25.

8. NeuschwanderTetri BA, Chaldwell SH. Nonalcoholic steatohepatitis: summary of an AASLD Single Topic Conference. Hepatology. 2003;37(5):1202-19.

9. Farrell GC, Larter CZ. Nonalcoholic fatty liver disease: fromsteatosis to cirrhosis. Hepatology. 2006;43:S99-S112.

10. Singh SP, Nayak S, Swain M, Rout N, Mallik RN, Agrawal O, et al. Prevalence of nonalcoholic fatty liver disease in coastal eastern India: a preliminary ultrasonographic survey. Trop Gastroenterol.2004;25:76-9.

11. Bajaj S, Nigam P, Luthra A, Pandey RM, Kondal D, Bhatt SP, et al. A case-control study on insulin resistance, metabolic co-variates \& prediction score in non-alcoholic fatty liveSr disease. Indian J Med Res. 2009;129(3):285-92.

12. Clark JM. The epidemiology of nonalcoholic fatty liver disease in adults. J Clin Gastroenterol. 2006;40:5-10.

13. Leite NC, Salles GF, Araujo AL, Villela-Nogueira CA, Cardoso $C R$, et al. Prevalence and associated factors of nonalcoholic fatty liver disease in patients with type diabetes mellitus. Liver Int. 2009; 29(1):113-9.

14. Lizardi-Cervera J, Laparra DI, Chávez-Tapia NC, Ostos ME, Esquivel MU. Prevalence of NAFLD and metabolic syndrome in asym-tomatics subjects. Rev Gastroenterol Mex. 2006;71(4):4539.

15. Jin $\mathrm{H}, \mathrm{Gu} Z, \mathrm{Yu} C$, Li Y. Association of non-alcoholic fatty liver disease with type 2 diabetes: clinical features and independent risk factors in diabetic fatty liver patients. Hepatobiliary Pancreat Dis Int. 2005;4:389-92.

16. Toledo FG, Sniderman AD, Kelley DE. Influence of hepatic steatosis (fatty liver) on severity and composition of dyslipidemia in type 2 diabetes. Diabetes Care. 2006;29:1845-50.

17. Raz I, Eldor R, Cernea S, Shafrir E. Diabetes: insulin resistance and derangements in lipid metabolism. Cure through intervention in fat transport and storage. Diabetes Metab Res. 2005;21:3-14.

18. Duseja A, Das A, Das R, Dhiman RK, Chawla Y, Bhansali $A$, et al. The Clinic opathological Profile of Indian Patients with Nonalcoholic Fatty Liver Disease (NAFLD) is Different from that in theWest. Dig Dis Sci. 2007;52:2368-74.

19. Bajaj S, Nigam P, Luthra A, Pandey RM, Kondal D, Bhatt SP, et al. A case-control study on insulin resistance, metabolic covariates\& prediction score in non-alcoholic fatty liver disease. IndianJ Med Res 2009;129:285-92.

20. Obika M, Noguchi H. Diagnosis and evaluation of nonalcoholic fatty liver disease. Exp Diabetes Res. 2012;2012:145754. doi:10.1155/2012/145754.

21. Caldwell SH, Harris DM, Patrie JT, Hespenheide EE.Is NASH under diagnosed among African American? Am J Gasteroenterol 2003; 98: 9607. 